Service social

\title{
Les orientations de recherche d'aide chez les aînés regroupés
}

\section{Marc-André Delisle et Hector Ouellet}

Volume 49, numéro 1, 2002

URI : https://id.erudit.org/iderudit/006876ar

DOI : https://doi.org/10.7202/006876ar

Aller au sommaire du numéro

Éditeur(s)

École de service social de l'Université Laval

ISSN

1708-1734 (numérique)

Découvrir la revue

Citer cet article

Delisle, M.-A. \& Ouellet, H. (2002). Les orientations de recherche d'aide chez les aînés regroupés. Service social, 49(1), 1-34. https://doi.org/10.7202/006876ar

\section{Résumé de l'article}

Les orientations de recherche d'aide ont été vues au cours d'une étude menée auprès de 360 aînés. Une liste de 15 problèmes pouvant affecter les gens âgés a été proposée aux répondants à qui l'on a demandé de nommer la ressource la plus adéquate. Les orientations de recherche d'aide les plus souvent mentionnées sont l'orientation vers Soi-même, puis vers les Proches et vers les Services. Les corrélations simples entre les orientations montrent qu'elles sont distinctes sans être exclusives. Les gens privilégient un type de ressources, mais peuvent en solliciter d'autres. Les orientations de recherche d'aide ne sont donc pas tant déterminantes de la fréquence de l'utilisation des services que de la manière dont les usagers les abordent. 


\title{
Les orientations de recherche d'aide chez les aînés regroupés ${ }^{1}$
}

\author{
Marc-André DELISLE \\ Hector OUELLET
}

Les orientations de recherche d'aide ont été vues au cours d'une étude menée auprès de 360 aînés. Une liste de 15 problèmes pouvant affecter les gens âgés a été proposée aux répondants à qui l'on a demandé de nommer la ressource la plus adéquate. Les orientations de recherche d'aide les plus souvent mentionnées sont l'orientation vers Soi-même, puis vers les Proches et vers les Services. Les corrélations simples entre les orientations montrent qu'elles sont distinctes sans être exclusives. Les gens privilégient un type de ressources, mais peuvent en solliciter d'autres. Les orientations de recherche d'aide ne sont donc pas tant déterminantes de la fréquence de l'utilisation des services que de la manière dont les usagers les abordent.

Mots clés : Orientations d'aide, utilisation des services, soutien social, participation sociale, personnes âgées

In a study conducted over 360 elderly group members, we have asked the respondents to identify most appropriate sources of help to solve problems (from a list of 15 problems) which could typically concern them. Self-reliance is the most frequently mentioned orientation, followed by orientation toward relatives and services. Correlation matrix between the orientations suggests that they are distinct but not exclusive. People seem to follow their usual pattern of help-seeking orientation but could go toward alternate resources. The help-seeking orientations would then less determine the frequency of the use of services than the way these services are used.

Key words : Service use, Social support, Social participation, Elderly

1 Ce projet de recherche a été réalisé grâce à une subvention du ministère de la Santé et des Services sociaux du Québec et de la Régie régionale ChaudièreAppalaches dans le cadre du Programme de subventions en santé publique. La rédaction de cet article a pu être complétée grâce à des fonds des comités de perfectionnement des chargés de cours de l'Université Laval et de I'Université du Québec à Chicoutimi. 


\section{INTRODUCTION}

Cet article porte sur les orientations de recherche d'aide chez les gens âgés. La pertinence de cette question tient au fait que le vieillissement démographique est susceptible d'entraîner une augmentation des besoins de soutien de la part des gens âgés et que, pour répondre à cette demande, la société devra se réorganiser (Lux, 1995). Dès lors, il importe de définir les orientations de recherche d'aide des aînés et de connaître les attributs des personnes axées sur chaque type de ressources.

Dans cet article, nous livrons les résultats d'une étude exploratoire qui aborde ce sujet. D'abord, seront exposés les aspects théoriques de l'étude, puis sa méthodologie et, ensuite, ses principaux résultats. En conclusion, nous en dégagerons les implications théoriques et pratiques.

\section{ASPECTS THÉORIQUES, RECENSION DES ÉCRITS ET HYPOTHÈSES}

Les principaux concepts utilisés dans l'étude sont ceux d'orientation de recherche d'aide, de recours aux services sociaux et de santé, ainsi que d'attributs des orientations de recherche d'aide. Les orientations de recherche d'aide sont des prédispositions au recours à des formes de soutien données. Elles sont analogues mais non identiques aux attitudes et aux habitus. Selon Lafrenaye (1994), l'attitude est un " état d'esprit » qui amène l'individu à répondre d'une façon particulière à des stimuli spécifiques. L'attitude n'est pas un comportement, bien qu'elle puisse en susciter quand le contexte s'y prête. De plus, l'attitude « comporte des composantes cognitive (croyances), affective (émotions) et comportementale (intentions d'agir) » (ibid., p. 402). Aussi, elle est orientée vers un objet et elle a une intensité.

Il en va de même des orientations de recherche d'aide. Sur le plan cognitif, ces orientations renvoient à la connaissance des ressources vers lesquelles la personne se tourne quand elle est en difficulté. En effet, l'individu orienté vers une ressource devrait mieux la connaître que la personne axée sur d'autres formes d'aide. La dimension affective des orientations de recherche d'aide a trait à l'investissement émotionnel de la part des gens. À 
la différence de l'attitude, nous croyons que l'investissement affectif dans une orientation de recherche d'aide ressortit davantage à la signification que celle-ci prend, donc à sa dimension symbolique, qu'aux sentiments éprouvés pour la ressource en question. Ainsi, se débrouiller seul (orientation vers soi-même) symbolise la conformité à un modèle culturel valorisé : l'autonomie ou le self-reliance. Ce type d'orientation est affectivement investi pour ce qu'il représente et non pour ce qu'il est, tandis que l'objet de l'attitude (être ou chose) est aimé ou détesté pour lui-même. Au reste, les orientations de recherche d'aide ont une intensité et tendent à l'invariance, quoiqu'elles puissent changer au gré des circonstances. Enfin, comme les attitudes, les orientations de recherche d'aide sont des prédispositions à l'action et non des comportements.

Le caractère symbolique des orientations de recherche d'aide rapproche ce phénomène de celui de l'habitus. Dans la perspective de Bourdieu (1984, p. 133-137), l'habitus est un « système de dispositions [inconscientes et durables] acquises par l'apprentissage implicite ou explicite qui fonctionne comme système de schémas cognitifs et corporels $»$. Ces dispositions sont le " produit de l'intériorisation des structures du monde social » et " des valeurs du groupe » (Durand et Weil, 1997, p. 264-265; voir aussi Lalive d'Épinay, 1989). Pour désigner cette dimension particulière de l'habitus, Bourdieu parle d'ethos [de classe], concept qu'il a emprunté à Weber. II en va ainsi des orientations de recherche d'aide: elles proviennent des groupes auxquels appartient la personne : groupes de sexe; famille et communauté; classe socioéconomique et culturelle, etc. Les individus les acquièrent et les intériorisent, ce qui les amène à réagir de manière analogue dans les mêmes situations.

Le concept de services sociaux et de santé rend compte des diverses formes d'aide données par des professionnels des secteurs public ou privé (d'après Zay, 1981, p. 495). Tel qu'il est employé ici, ce concept exclut le soutien fourni par les aidants naturels. Par contre, il englobe les services qui ont trait à la santé physique (services médicaux et paramédicaux) et mentale (psychosociaux) de la personne, ceux qui ont un caractère économique (soutien du revenu), et ceux qui touchent au maintien à domicile, au logement, à l'hébergement, au transport et au loisir. 
Les notions de recours aux services et d'utilisation des services renvoient aux comportements des gens qui entrent en rapport avec les intervenants qui fournissent ces services. Dans cet article, ces deux termes sont souvent employés comme synonymes. Toutefois, le concept de recours aux services est plus large que celui d'utilisation. Ce dernier se limite à la consultation des professionnels de la santé et des services psychosociaux ainsi qu'au fait de demander et de recevoir des services de la part des organismes qui en donnent. À cela s'ajoutent, dans la notion de recours aux services, les démarches entreprises pour connaître leur accessibilité. Le fait de s'informer sur les services est considéré comme un comportement révélateur de besoins réels ou anticipés, donc préalable à leur utilisation.

Précisons enfin que nous étudions les attributs des personnes axées sur les orientations de recherche d'aide et non les déterminants de ces orientations. Les attributs sont des facteurs associés aux orientations de recherche d'aide qui peuvent leur être antécédents ou conséquents. Comme on le verra plus loin, le sexe et le statut socioéconomique sont des antécédents des orientations de recherche d'aide, alors que l'utilisation des services en est une conséquence possible. L'ensemble de ces facteurs constituent les attributs des personnes axées sur les différentes orientations de recherche d'aide.

Celles-ci n'ont pas été étudiées systématiquement, du moins à notre connaissance. Dès lors, pour fonder des hypothèses susceptibles d'expliquer les attributs des personnes axées sur les orientations de recherche d'aide, il a fallu puiser à la fois dans la documentation portant sur les comportements de recherche d'aide (help-seeking behavior) et sur celle qui a trait à l'utilisation des services.

Un premier examen de cette littérature montre qu'en amont des orientations de recherche d'aide et des comportements qui en dérivent il y a des facteurs socioéconomiques comme les conditions et le mode de vie des gens, des variables socioculturelles comme les valeurs auxquelles ils adhèrent ainsi que des caractéristiques personnelles (Logan et Spitze, 1994; McCaslin, 1989; McKinlay, 1972). En aval de ces orientations, il y a des facteurs contextuels comme l'acuité des problèmes rencontrés et 
l'accessibilité des ressources, facteurs déterminants de l'utilisation ou de la non-utilisation des ressources sur lesquelles les gens sont axés. De sorte qu'une même orientation de recherche d'aide pourra se traduire par le recours à une pluralité de ressources ou par leur non-utilisation (McCaslin, 1989, p. 170).

De plus, la documentation sur l'utilisation des services sociaux et de santé montre que celle-ci n'est pas uniquement déterminée par les besoins (Mitchell et Krout, 1998; Tijhuis, Peters et Foets, 1990; Wan, 1989; Wolinsky et Johnson, 1991). Des individus défavorisés demandent peu de services, alors que d'autres, mieux nantis ou en meilleure santé, y ont davantage recours. Une explication de ce phénomène veut que certains groupes sociaux soient portés à utiliser les services parce que ce comportement fait partie de leur mode de vie et de leur culture, donc parce qu'ils sont orientés vers les services. C'est le cas des adultes de classe moyenne et supérieure, de ceux qui résident en milieu urbain et des plus scolarisés (Lebel, 1986; Weinert et Long, 1987). Les aînés plus riches et plus instruits se sentent davantage à l'aise avec le fonctionnement de la structure de services et savent mieux comment composer avec ses agents que les plus démunis (Cho, 1998; McKinlay, 1972; Moon, Lubben et Villa, 1998). À l'inverse, la plupart des « sous-consommateurs » de services appartiennent aux groupes sociaux étrangers à l'univers socioculturel de ceux qui les livrent (Cho, 1998; Keating, 1991; McKinlay, 1973; Moen, 1978; Moon, Lubben et Villa, 1998; Ouellet, 1993). En plus d'avoir des valeurs différentes quant à l'utilisation des services, ces derniers vivent une forme d'aliénation culturelle par rapport aux dispensateurs de services qui les incite à ne pas demander de services sauf en cas de grande nécessité (McKinlay, 1972, p. 125).

Il y aurait donc un ethos du recours aux services en vertu duquel, pour certains, par exemple, il serait « de bon ton » de passer des examens médicaux préventifs alors que d'autres se priveraient de soins élémentaires. Cet ethos est un ensemble de normes qui dérivent des valeurs du groupe de référence de l'individu et qui sont au fondement de la logique sociale sous-jacente aux rapports avec les services (Delisle, 1989). II est tributaire de la distance entre l'univers des clients et celui des dispensateurs de services. Plus la distance est faible, plus les gens sont orientés vers les 
services, et inversement. La distance est fonction des caractéristiques socioéconomiques et culturelles (revenu, scolarité, valeurs, etc.) des receveurs et des dispensateurs de services. Elle est sans doute mesurable, mais nous ne l'avons pas évaluée systématiquement dans notre étude. Nous l'avons estimée de façon sommaire à partir des caractéristiques des répondants et de la connaissance que nous avons de celles des dispensateurs de services.

De plus, les hommes et les femmes n'ont pas des orientations identiques par rapport aux services, les hommes étant plus portés à se débrouiller seuls que les femmes (Dulac, 1997; Penning et Strain, 1990; Tijhuis, Peters et Foets, 1990). Certaines études laissent croire qu'il n'y a pas de différence entre les sexes en ce qui a trait aux services d'entretien domestique, aux soins à domicile et aux soins médicaux d'urgence; d'autres recherches indiquent que les hommes se tournent davantage vers les ressources informelles (famille, parenté) pour leur entretien personnel ainsi que pour les travaux domestiques (Mitchell et Krout, 1998; Wan, 1989; Wolinsky et Johnson, 1991). Aussi, la socialisation des hommes les incite à nier l'importance des symptômes ressentis, alors que les femmes leur accordent plus d'attention (Dulac, 1997; Mutran et Ferraro, 1988). Les orientations de recherche d'aide des hommes et des femmes dériveraient donc de la manière dont chacun des groupes de sexe réagit "normativement" face aux difficultés de la vie, cette manière étant un ethos (de sexe ou de genre) transmis par les différentes formes de socialisation.

Ce n'est pas tout. Les personnes âgées des régions rurales utilisent moins souvent les services de santé physique et mentale que les aînés des grandes villes même lorsqu'elles éprouvent des difficultés analogues (Coward, 1988; Keating, 1991; Mitchell et Krout, 1998; Wan, 1989; Wolinsky et Johnson, 1991). Le fait que les services sociaux et de santé soient moins accessibles à la campagne qu'en ville (Keating, 1991) n'explique pas totalement ce phénomène, car le rapport aux services dépend aussi de la culture des usagers potentiels (Corin, 1984; Coward, 1988). Les ruraux âgés contemporains ne sont pas habitués à demander de l'aide à des personnes extérieures à leur milieu. Ils ont recours aux services après avoir parlé de leurs difficultés avec une 
personne de confiance qui sert de relais entre eux et l'organisme ou le professionnel.

Par ailleurs, comme l'a montré Corin (Corin, 1984; Corin, Sherif et Bergeron, 1983), devant un problème, la personne âgée peut choisir de ne rien faire, tenter de se débrouiller seule, demander de l'aide à ses proches, aller vers les services sociaux et de santé ou solliciter plusieurs types de ressources simultanément. Ces réactions correspondent aux différentes orientations de recherche (ou de non-recherche) d'aide.

Certains auteurs parlent d'un « modèle hiérarchique compensatoire " (Hierarchical compensatory model of social support) en vertu duquel la personne qui cherche une solution à ses problèmes tenterait de se débrouiller seule, puis solliciterait l'aide de ses proches et ensuite les ressources formelles (Cantor, 1979 et 1991; Cantor et Little, 1985; Penning, 1990; Stoller, 1989). Le recours à des gens étrangers à la famille aurait alors un caractère compensatoire. II existe une contrepartie à ce modèle qui correspond à la théorie des fonctions partagées de Litwak (1989) et à ce que d'aucuns ont appelé le « modèle de la spécificité des tâches » (Task specificity model; voir Penning, 1990). Ces théories veulent que les aidants naturels et les aidants professionnels jouent des rôles distincts auprès des personnes âgées.

Les études effectuées pour vérifier les fondements empiriques de ces modèles théoriques les accréditent partiellement. D'une part, comme le montre Penning (1990), l'existence du modèle hiérarchique compensatoire est corroborée par le fait que les aînés en difficulté ont tendance à aller vers les services quand les ressources informelles ne peuvent combler leurs besoins. Cependant, tous n'ont pas recours aux services et il n'y a pas nécessairement substitution des formes d'aide. D'autre part, les services rendus par les amis, les voisins, les membres de groupes communautaires et les organisations formelles ne remplacent pas ceux que donnent le conjoint, les enfants et les membres de la parenté (Chappell, 1985; Penning, 1990; Stoller, 1989). Par conséquent, les services rendus par les diverses catégories de personnes seraient plus complémentaires que compensatoires. 
Cela expliquerait les rapports quelque peu ambigus entre le soutien social et le recours aux services. En effet, ce sont habituellement les personnes les plus isolées ou soutenues de façon inadéquate qui utilisent le plus les services sociaux et de santé (Auslander et Litwin, 1990; Chappell, 1985; Wolinsky et Johnson, 1991). Les dispensateurs de services prennent alors la relève du réseau de soutien. À l'opposé, il y a des aînés bien entourés qui utilisent peu les services sociaux et de santé, parce que leur réseau comble l'essentiel de leurs besoins (Auslander et Litwin, 1990; Chappell, 1985). Mais dans certains milieux les aînés les mieux entourés ont davantage recours aux services, parce que leurs aidants servent de relais entre les personnes âgées et les ressources formelles (Logan et Spitze, 1994; Mitchell et Krout, 1998; Stoller, 1989; Wolinsky et Johnson, 1991).

Enfin, certaines variables concomitantes sont susceptibles d'influencer les orientations de recherche d'aide et leur traduction en demande de soutien. C'est le cas de la participation et de la cohésion sociales, des contacts sociaux, de l'âge et de la scolarité.

La participation à un groupe produit un " effet de normalisation » qui module les comportements de ses membres (Blau, 1960a et $1960 b)$. Les individus qui participent à un groupe ont en effet tendance à se conformer à ses normes et à se comporter de façon similaire par rapport à un objet donné (Guimond, 1994). La participation à un groupe pourrait donc entraîner une convergence dans les orientations relatives à l'utilisation des services.

Les effets structurants et normatifs du groupe seraient euxmêmes fonction de la cohésion entre ses membres. Plus le groupe est cohésif, c'est-à-dire plus les liens entre ses membres sont forts, plus il aurait de l'influence sur les attitudes et sur les comportements individuels, y compris ceux qui ont trait au recours aux services (Guimond, 1994; Lasnier, 1989; Mudrack, 1989). Ainsi se dégageraient des divers groupes des patterns favorisant soit la restriction dans le recours aux services, soit leur utilisation extensive ou, encore, le recours à des services spécifiques.

L'effet des contacts sociaux sur les orientations de recherche d'aide dépend des orientations valorisées dans le milieu 
fréquenté. Plus la personne a de relations sociales, plus elle subit l'influence de son entourage sous ce rapport. Cette influence peut jouer dans le sens d'une plus forte ou d'une plus faible orientation vers les services.

Enfin, les aînés plus jeunes connaissent mieux les services que les plus vieux (Calsyn et Roades, 1993; Moon, Lubben et Villa, 1998). Or, les "jeunes aînés » sont plus scolarisés que leurs ascendants (Gauthier et Duchesne, 1991). L'effet de l'âge sur la connaissance des services pourrait alors s'expliquer par la scolarité.

En résumé, la documentation consultée montre que les orientations de recherche d'aide sont des prédispositions à l'action dont les attributs tiennent à l'ethos de l'entourage des gens et à des caractéristiques personnelles qui renforcent ou atténuent l'influence du milieu. Les individus adoptent une orientation de recherche d'aide privilégiée sous l'influence de leur groupe d'appartenance. Au fil des ans, cette orientation devient une composante de leur identité sociale et module leurs comportements devant les problèmes rencontrés. De cette recension d'écrits, on peut déduire les hypothèses suivantes :

$\mathrm{H}_{1}$ Les personnes fortement orientées vers elles-mêmes dans la solution de leurs problèmes sont issues d'un milieu et d'une culture qui valorisent l'autonomie personnelle, c'est-à-dire la débrouillardise ou encore l'individualisme ${ }^{2}$. Par conséquent, on peut s'attendre à ce que ces personnes s'intéressent peu aux services et qu'elles soient moins portées à les utiliser.

$\mathrm{H}_{2}$ Les individus orientés vers leurs proches dans la solution de leurs problèmes sont issus de deux types de milieux : a) une collectivité réfractaire aux services; b) un réseau social

Ce double sens que peut prendre le concept d'autonomie a été évoqué avec justesse par l'un des réviseurs de l'article. Malheureusement, le caractère exploratoire de notre étude n'a pas permis de savoir à quel type d'autonomie se réfèrent les gens orientés vers eux-mêmes. Rien n'exclut qu'on y retrouve à la fois des " débrouillards » et des " individualistes ». Le cas échéant, l'orientation vers soi-même n'aurait pas la même signification pour chacune des deux catégories de personnes. $\mathrm{Ce}$ point demande à être vérifié par de futures recherches. 
favorable aux services, mais incapable de fournir un soutien adéquat à ses aînés. Le premier type de milieu favorise une sous-utilisation des services, alors que le second aide ses membres à les obtenir. Dans un échantillon qui regrouperait autant d'individus des deux milieux, les effets favorables et défavorables au recours aux services s'annuleraient et il n'y aurait pas de corrélation entre l'orientation vers les proches et le recours aux services.

$\mathrm{H}_{3}$ Enfin, les gens orientés vers les services viennent d'un milieu socioéconomique et culturel proche des personnes qui les dispensent. II est donc prévisible que ces gens connaissent bien les services et qu'ils les utilisent en cas de besoin.

\section{MÉTHOdOLOGIE}

\section{Échantillon}

La population à l'étude était celle des aînés de 50 ans et plus demeurant à domicile sur la rive-sud de Québec, qui participaient aux activités d'un des 16 organismes d'aînés les plus représentatifs de cette région en $1996^{3}$. L'échantillon a été tiré des listes de membres de ces organismes. II y avait aussi des personnes qui fréquentaient la Maison des aînés de Lévis, un centre communautaire qui était le partenaire principal de l'étude. L'échantillon est donc partiellement aléatoire, puisqu'il est composé d'individus choisis au hasard et de volontaires ${ }^{4}$. Un tel échantillon est considéré comme valable pour la mesure des relations entre les variables (Beaud, 1998) ${ }^{5}$.

II s'agissait du territoire du CLSC Chutes-de-la-Chaudière-Desjardins de 1996.

$4 \quad$ On trouvera une description détaillée de la façon dont cet échantillon a été tiré dans Delisle et Ouellet, 2001.

5 Lawton, Moss, Hoffman, Grant, Have et Kleban (1999) ont eu recours au même type d'échantillon dans leur étude. 


\section{Questionnaire et instruments de mesure}

Le questionnaire comprend des questions posées dans des recherches antérieures (Béland, 1982; Bolduc, 1986; Santé Québec, 1995) et des instruments construits spécifiquement pour l'étude. Afin d'estimer l'importance des orientations de recherche d'aide, nous avons dressé une liste de quinze problèmes qui touchent fréquemment les aînés. Pour chacun de ces problèmes, nous avons demandé aux répondants d'indiquer la ressource qui leur semblait la plus adéquate pour les gens de leur âge (question projective). Les six catégories de ressources mutuellement exclusives suggérées furent: soi-même; les enfants, les parents et les amis; les ressources communautaires; les professionnels du CLSC; les ressources médicales; un employé ou un professionnel privé. En comptant le nombre de fois où chacune des ressources a été mentionnée, nous avons créé les trois variables d'orientation de recherche d'aide utilisées par la suite, soit l'orientation vers Soi-même, l'orientation vers les Proches (enfants, parents et amis) et l'orientation vers les Services (ressources communautaires + professionnels du CLSC + médecin [échelles théoriques 0 à +15$])^{6}$. À cela s'ajoute l'orientation Ne sait pas, c'est-à-dire le nombre de fois où la personne a dit ne pas savoir à qui s'adresser quand elle est aux prises avec une difficulté particulière. La même liste de problèmes a servi à la construction de la variable nombre de Problèmes rencontrés qui a été élaborée en comptant ceux auxquels le répondant a déjà été confronté (échelle identique).

À partir des items du questionnaire, nous avons construit un ensemble de variables susceptibles d'être antécédentes aux orientations de recherche d'aide. C'est le cas des caractéristiques démographiques et socioéconomiques usuelles: l'âge (50 à 89 ans), le sexe (femme=0; homme=1), l'état civil (non marié=0; marié=1), la situation de ménage (seul=0; vit avec d'autres=1), le revenu, la scolarité ainsi que la santé perçue et la santé mentale. L'indice de Santé perçue utilisé dans cette étude est le même que celui de l'Enquête Santé Québec de 1995 (échelle 0 [mauvaise] à +4 [excellente]). Quant à la mesure de la Santé mentale, c'est un indice d'« Inconfort » psychologique employé dans d'autres

6 Dans ce texte, nous avons mis des majuscules au nom des variables considérées. 
recherches (échelle 0 [inconfort maximal] à +15 [confort maximal]). Cet instrument a une forte consistance interne, puisque son alpha de Cronbach est de 0,82 dans cette étude et dans celle de Portneuf (Delisle, Boucher et Roy, 1993), et de 0,85 dans Lotbinière (Simard, Delisle, Drouin et Larouche, 1992).

Nous avons considéré plusieurs indicateurs de soutien social, soit : le nombre d'Aidants disponibles en cas d'urgence (échelle 0 à +5 ), le nombre de Services rendus à des membres de groupes d'aînés et reçus de leur part (échelle 0 à +22 ), la fréquence des Contacts sociaux (échelle 0 à +60 ; alpha de Cronbach : 0,75 ), et le nombre de Groupes d'appartenance (échelle 0 à +7 ). II y a également les variables collectives de Participation et de Cohésion sociales dont le mode d'élaboration a été décrit de façon détaillée dans un autre document ${ }^{7}$. La Participation sociale a été définie opérationnellement comme étant la fréquence des activités organisées par le groupe d'appartenance du sujet et les groupes ayant participé à l'étude ont été classés selon ce critère. Après quoi, leurs membres se sont vu attribuer la cote de participation de celui-ci (faible [0] ou fort [1]). La Cohésion du groupe se manifeste par l'attraction interpersonnelle, par l'attachement au groupe et par l'attrait que le groupe exerce sur ses membres (Lasnier, 1989). Elle est mesurée par un instrument de recherche développé pour les jeunes, que nous avons adapté pour les aînés. Nous avons classé les groupes qui ont participé à l'étude selon l'indice de cohésion de leurs membres, à qui nous avons ensuite attribué la cote de cohésion de leur groupe (faible [0] ou fort [1]). Plusieurs tests statistiques (différences de moyennes et analyses discriminantes) ont permis de valider ces échelles.

Nous avons aussi tenu compte de la Circulation de l'information sur les services (échelle 0 à +4 ). De même, nous avons créé une variable en additionnant les réponses à deux questions portant sur la fréquence à laquelle les gens Discutent de leurs problèmes personnels et de santé avec des membres de groupes d'aînés (échelle 0 à +6 ). 
Les variables de recours aux services ont été considérées comme des conséquences des orientations de recherche d'aide. Parmi ces variables, il y a le nombre d'Organismes de services sociaux et de santé connus (échelle 0 à +12 ), variable qui exclut la Maison des aînés de Lévis. Viennent ensuite les services utilisés au cours des six mois ayant précédé la recherche, soit: le nombre d'Organismes de services contactés (échelle 0 à +12 ), les Consultations médicales préventives (échelle $0+30$ ) et les Consultations médicales curatives (même échelle) ${ }^{8}$.

\section{Collecte et traitement des données}

Les données ont été recueillies au printemps 1996 à l'occasion d'activités organisées par la Maison des aînés de Lévis. Au total, 360 questionnaires ont été remplis et 358 ont été conservés pour analyse. L'échantillon obtenu a été comparé à la population âgée du Québec de 1991 et à un échantillon représentatif des membres de la Fédération de l'âge d'or du Québec (FADOQ, 1993). Les orientations de recherche d'aide ont d'abord été étudiées de façon descriptive. Puis nous avons regardé les corrélations simples entre les variables susceptibles d'être des antécédents ou des conséquences de chacune des orientations de recherche d'aide (vers Soi-même, vers les Proches ou vers les Services).

Afin obtenir des données plus précises sur les attributs des personnes axées sur chaque type de ressources, nous avons réparti ces personnes en trois classes: les individus faiblement, modérément et fortement orientés vers les différentes catégories

Le texte du questionnaire était explicite en ce qui a trait à la distinction entre les consultations faites à titre préventif ou à titre curatif. Les consultations préventives étaient décrites de la façon suivante: $Q$ 52. Au cours des six derniers mois, combien de fois avez-vous consulté l'un ou l'autre des professionnels suivants à titre préventif, c'est-à-dire uniquement pour obtenir des renseignements ou pour savoir si vous étiez en bonne santé (sans être souffrant/e ni malade ni blessé/e)? Quant aux consultations curatives, le questionnaire les décrivait ainsi: $Q$ 53. Au cours des six derniers mois, combien de fois avez-vous consulté l'un ou l'autre des professionnels suivants pour obtenir des soins ou des services de leur part? Les variables construites à partir de ces questions représentent la somme de la fréquence des consultations avec chacune des catégories de professionnels considérés, c'est-à-dire le nombre de professionnels multiplié par la fréquence de consultation de chacun d'eux. 
de ressources. Les personnes faiblement orientées vers chaque type de ressources représentent $29,9 \%$ de l'échantillon total pour l'orientation vers Soi-même, $31 \%$ pour l'orientation vers les Proches et $27,9 \%$ pour l'orientation vers les Services. Les individus modérément orientés vers chacune des ressources forment respectivement $41,3 \%, 38,5 \%$ et $38,8 \%$ de l'échantillon total. Enfin, les individus fortement orientés vers ces mêmes ressources constituent $28,8 \%$ de l'échantillon total pour l'orientation vers Soi-même, 30,4\% pour l'orientation vers les Proches et $33,2 \%$ pour l'orientation vers les Services. Comme nous voulions connaître les attributs des gens les plus faiblement et les plus fortement orientés vers les ressources, nous avons éliminé les individus qui étaient modérément axés sur elles. Après quoi, nous avons effectué des analyses discriminantes en opposant les groupes faiblement et fortement orientés vers chaque type de ressources (Tabachnik et Fidell, 1996). Nous avons entré dans ces analyses uniquement les variables corrélées de façon significative (au niveau bivarié) aux orientations de recherche d'aide, en tenant pour acquis que les variables qui n'étaient pas corrélées ne pouvaient être des attributs majeurs des personnes axées sur les différentes ressources. Nous présentons les résultats des analyses effectuées en indiquant toutes les statistiques significatives à $p<0,05$. Cependant, considérant la pluralité des tests statistiques, on accordera davantage de crédibilité à ceux dont le niveau de signification est le plus bas.

\section{RÉSULTATS}

\section{Caractéristiques sociodémographiques}

Plus du tiers de l'échantillon $(36,4 \%)$ est formé de personnes reliées à la Maison des aînés de Lévis et aux clubs de l'âge d'or qui utilisent ses équipements, mais qui ne s'identifient pas à cet organisme. Dans l'échantillon, la proportion de jeunes aînés (5064 ans) est analogue à celle qu'on retrouve dans les clubs de l'âge d'or du Québec (FADOQ, 1993), soit environ le tiers des participants. Le pourcentage de gens de 75 ans et plus par rapport aux 65 ans et plus est plus grand dans l'échantillon (35,7\%) que chez les membres de la FADOQ $(21,2 \%)$, mais il ne l'est pas plus que dans la population de 65 ans et plus du Québec, soit 38,7 \% (Québec [Prov.], 1993). Quant aux proportions d'hommes 
et de femmes dans l'échantillon total (32,5\%-67,5\%), elles sont presque identiques à celles de la FADOQ (34\%-66\%). Ces données montrent que l'échantillon de l'étude est à l'image de sa population d'origine même s'il n'est pas systématiquement aléatoire.

\section{Problèmes rencontrés}

Les quinze problèmes les plus fréquemment rencontrés par les répondants sont: le besoin de conseils sur l'impôt et la succession (33,6\%), l'inquiétude par rapport à la santé $(27,7 \%)$, l'entretien de l'extérieur du domicile $(22,8 \%)$, l'inquiétude par rapport aux enfants et au conjoint $(22,1 \%)$, l'entretien de l'intérieur du domicile $(19,2 \%)$, l'ennui, la solitude et l'isolement $(17,6 \%)$, le transport $(12,7 \%)$, l'inquiétude par rapport à la sécurité $(11,1 \%)$, les difficultés financières $(8,1 \%)$, les relations avec le conjoint $(6,5 \%)$, les relations avec les enfants $(6,5 \%)$, la préparation des repas $(5,5 \%)$, l'hygiène personnelle $(4,6 \%)$, l'exploitation ou les abus de la part de l'entourage $(3,6 \%)$, et les problèmes avec le propriétaire ou les locataires (2,6\%). Le nombre moyen de pro-blèmes rencontrés est de 1,75 par individu. Un peu plus du tiers $(35,8 \%)$ des répondants ne mentionnent aucun problème; $24,9 \%$ en nomment un seul, et les autres (près de $40 \%$ ) doivent faire face à deux problèmes ou plus. Donc, la majorité des sujets de l'étude ne sont pas accablés par de multiples problèmes, résultat qui corrobore les observations de Corin, Sherif et Bergeron (1983, p. 200).

\section{Ressources privilégiées}

Le tableau 1 présente les catégories de ressources privilégiées par les répondants pour résoudre leurs problèmes. Ce tableau met en évidence deux phénomènes: tout d'abord, l'importance accordée à l'autonomie individuelle et, en second lieu, le « modèle hiérarchique compensatoire " d'utilisation des ressources. D'une part, la primauté de l'autonomie individuelle ressort nettement: la ressource soi-même est la plus souvent mentionnée, ce qui corrobore les observations de Moen (1978), de Paquet (1996) et de Tremblay (1999) sur la réticence de certains aînés et de leurs proches à demander de l'aide. 
D'autre part, selon les répondants, les aînés confrontés à des problèmes qu'ils ne peuvent résoudre devraient d'abord faire appel à leurs proches, puis aux ressources communautaires, aux professionnels du CLSC, au médecin ou à un employé. Dans la mesure où un principe de substitution opère ici, il pourrait correspondre au modèle hiérarchique compensatoire décrit par Cantor (1979 et 1991). Cependant, telles que furent posées les questions (de façon projective), il est impossible d'affirmer que les différentes ressources se substituent les unes aux autres dans l'ordre où elles furent présentées, car rien ne dit qu'un aîné continue de chercher de l'aide tant qu'il n'en a pas obtenu, et qu'il se tourne vers d'autres ressources lorsque celle qu'il privilégie n'est pas disponible. D'autant plus qu'au total les services (ressources communautaires + CLSC + médecin) sont davantage privilégiés que les proches. 


\section{Tableau 1}

Orientations de recherche d'aide. Nombre moyen de mentions de chacune des ressources par répondant

\begin{tabular}{|l|c|}
\hline & Moyenne \\
\hline Soi-même & 4,9 \\
\hline Enfants, parents, amis (proches) & 2,1 \\
\hline & \\
\hline Ressources communautaires & 1,5 \\
\hline CLSC & 1,37 \\
\hline Médecin & 0,8 \\
\hline & \\
\hline $\begin{array}{l}\text { Ensemble des services (ressources } \\
\text { communautaires + CLSC + médecin) }\end{array}$ & 3,7 \\
\hline & \\
\hline Employé & 0,71 \\
\hline & 1,22 \\
\hline Ne sait pas & 0,18 \\
\hline Plusieurs ressources & 358 \\
\hline n & \\
\hline
\end{tabular}

De plus, la matrice des corrélations simples entre les diverses orientations de recherche d'aide montre que chacune d'elles est distincte (tableau 2). Ainsi, l'orientation vers Soi-même est corrélée négativement mais significativement à toutes les autres, y compris à l'orientation Ne sait pas. Dès lors, plus une personne est orientée vers elle-même dans la solution de ses problèmes, moins elle est orientée vers d'autres ressources, ce qui est logique, et elle sait quoi faire lorsqu'elle est en difficulté. Quant à l'orientation vers les Proches, elle n'est corrélée à aucune autre orientation si ce n'est, de façon négative, à l'orientation vers Soimême. Par contre, les orientations vers les différents services (CLSC, ressources médicales et communautaires, employé) sont intercorrélées, ce qui donne à penser que, pour les répondants, les services peuvent être substituables les uns aux autres dans certaines circonstances. S'il existe un modèle hiérarchique compensatoire, ce serait donc seulement entre les services, parce que les résultats de cette recherche ne mettent pas en évidence de lien de continuité entre la ressource Soi-même, les Proches et les Services. 


\section{Tableau 2}

\section{Corrélations simples ( $r$ de Pearson) entre les orientations de recherche d'aide}

\begin{tabular}{|c|c|c|c|c|c|c|c|}
\hline & $\begin{array}{l}\text { Soi- } \\
\text { même }\end{array}$ & $\begin{array}{l}\text { Enfants, } \\
\text { parents, } \\
\text { amis }\end{array}$ & CLSC & Médecin & $\begin{array}{c}\text { Ressour- } \\
\text { ces } \\
\text { commu- } \\
\text { nautaires }\end{array}$ & Employé & $\begin{array}{c}\text { Ne sait } \\
\text { pas }\end{array}$ \\
\hline \multicolumn{8}{|l|}{ Soi-même } \\
\hline $\begin{array}{l}\text { Enfants, } \\
\text { parents, amis }\end{array}$ & $-0,30^{\star \star *}$ & & & & & & \\
\hline CLSC & $-0,45^{* * *}$ & 0,08 & & & & & \\
\hline Médecin & $-0,22^{\star \star \star}$ & $-0,03$ & $0,16^{* *}$ & & & & \\
\hline $\begin{array}{l}\text { Ressources } \\
\text { commu- } \\
\text { nautaires }\end{array}$ & $-0,43^{* * *}$ & 0,03 & $0,33^{\star * *}$ & $0,17^{* \star *}$ & & & \\
\hline Employé & $-0,19^{\star \star \star}$ & $-0,04$ & $0,13^{*}$ & $0,23^{* * *}$ & 0,03 & & \\
\hline Ne sait pas & $-0,19^{\star * *}$ & $-0,06$ & $-0,09$ & $-0,05$ & $-0,08$ & $0,15^{* *}$ & \\
\hline $\begin{array}{l}\text { Services } \\
\text { (ressources } \\
\text { communau- } \\
\text { taires + CLSC } \\
+ \text { médecin) }\end{array}$ & $-0,55^{\star \star \star}$ & 0,04 & nsp & nsp & nsp & $0,15^{\star *}$ & $-0,11^{*}$ \\
\hline
\end{tabular}

* $p<0,05^{* *} p<0,01{ }^{* * *} p<0,001$

- nsp : Ne s'applique pas, parce qu'il y a des indicateurs communs dans les variables considérées.

Mais les corrélations observées sont modérées ou faibles. On peut alors se demander si les orientations de recherche d'aide sont exclusives. Autrement dit, les gens ont-ils tendance à n'avoir recours qu'à un seul genre de ressources? Pour répondre à cette question, nous avons dichotomisé les orientations de recherche d'aide de manière à opposer ceux qui n'ont mentionné qu'un seul type de ressource à ceux qui en ont évoqué plusieurs. Le tableau 3 présente les combinaisons de ressources vers lesquelles les répondants sont orientés. Ces résultats indiquent que la majorité des répondants sont orientés vers une pluralité de ressources. Les orientations de recherche d'aide ne sont donc pas exclusives. Par conséquent, les gens se tournent vers la ressource qu'ils privilégient (et qui correspond à leur orientation principale ou dominante) chaque fois que cette ressource est accessible. Le cas échéant, ils envisagent une solution de 
rechange qui constitue une orientation subsidiaire acceptable à leurs yeux.

\section{Tableau 3}

Répartition des répondants en fonction de leurs orientations de recherche d'aide (\%)

a. Répartition par combinaison de ressources

\begin{tabular}{|l|c|}
\hline Soi, proches et services & 40,2 \\
\hline Soi et proches & 13,7 \\
\hline Soi et services & 13,7 \\
\hline Proches et services & 13,4 \\
\hline Soi seulement & 9,8 \\
\hline Services seulement & 4,7 \\
\hline Proches seulement & 1,7 \\
\hline Aucun, autre et/ou ne sait pas & 2,8 \\
\hline Total (\%) & 100 \\
\hline $\mathbf{n}$ & 358 \\
\hline
\end{tabular}

b. Répartition selon le genre de ressources

\begin{tabular}{|l|c|}
\hline Trois genres de ressources & 40,2 \\
\hline Deux genres & 40,8 \\
\hline Un genre & 16,2 \\
\hline Aucun, autre et/ou ne sait pas & 2,8 \\
\hline Total (\%) & 100 \\
\hline $\mathbf{n}$ & 358 \\
\hline
\end{tabular}

c. Répartition selon la ressource mentionnée (catégories non exclusives)

\begin{tabular}{|l|c|}
\hline Soi & 77,4 \\
\hline Proches & 69,0 \\
\hline Services & 72,0 \\
\hline Aucun, autre et/ou ne sait pas & 2,8 \\
\hline $\mathbf{n}$ & 358 \\
\hline
\end{tabular}

d. Répartition selon la ressource non mentionnée (catégories non exclusives)

\begin{tabular}{|l|c|}
\hline Soi & 22,6 \\
\hline Proches & 31,0 \\
\hline Services & 27,9 \\
\hline $\mathbf{n}$ & 358 \\
\hline
\end{tabular}


On peut aussi se demander si les répondants s'orientent vers les ressources les plus adéquates par rapport aux problèmes mentionnés. Certes, il est difficile d'évaluer l'adéquation des ressources lorsqu'elles sont polyvalentes comme les proches ou les services communautaires, mais certaines ressources semblent plus utiles au regard des problèmes évoqués.

Ainsi, pour l'entretien de l'extérieur du domicile, on se fie d'abord à soi-même $(39,4 \%)$, puis à un employé $(26 \%)$ et aux proches (23\%). Les mêmes ressources sont envisagées pour l'entretien de l'intérieur du domicile, sauf que la ressource Soi-même est plus souvent mentionnée (52,6\%), alors que les Proches le sont moins $(13,2 \%)$. Pour ces problèmes, les ressources communautaires se classent au quatrième rang avec $4,5 \%$ de mentions pour l'extérieur du domicile et $7,1 \%$ pour l'intérieur. Compte tenu de l'existence sur la rive-sud de Québec de services d'entraide bien organisés (Hamelin, 1995), ces pourcentages semblent faibles. Nous ne savons pas pourquoi les aînés n'ont pas parlé de ces services. Ils ne les connaissaient peut-être pas.

Lorsqu'on a des problèmes de transport, on s'organise par soimême (58 \%) ou l'on fait appel aux proches (24,9\%). Les ressources communautaires viennent au troisième rang avec seulement $8,3 \%$ de mentions. Pour l'hygiène personnelle, on se débrouille soi-même $(53,6 \%)$ ou on a recours aux professionnels du CLSC $(25,5 \%)$ et quelquefois aux proches $(8,4 \%)$. Pour la préparation des repas, on s'arrange soi-même $(61,4 \%)$ ou on fait appel aux ressources communautaires $(18,8 \%$ ) ou aux proches $(9,4 \%)$. En ce qui a trait à l'inquiétude par rapport à la santé, ce sont dans l'ordre : le médecin (47,3\%), soi-même $(23,2 \%)$ et les proches $(11,7 \%)$. On n'a pas souvent recours aux professionnels du CLSC dans de telles situations (seulement 9,5\% de mentions). Quand on est inquiet pour sa sécurité, on se fie à soi-même $(34,7 \%)$, aux proches $(24,8 \%)$ et aux ressources communautaires $(19,8 \%)$, mais rarement aux professionnels du CLSC (6,9 \% de mentions). L'inquiétude par rapport au conjoint et aux enfants amène les aînés à se fier à eux-mêmes $(31,1 \%)$, aux professionnels du CLSC (18,7\%), aux proches (16,7\%), ensuite aux ressources communautaires $(9 \%)$ et au médecin $(8,7 \%)$. En cas de difficultés financières, on s'organise soi-même $(40,1 \%)$ ou l'on fait appel aux proches $(16,1 \%)$ ou aux ressources 
communautaires $(13,4 \%)$. Quand on s'ennuie, on se tourne vers les proches $(31,2 \%)$, on se divertit soi-même $(27,8 \%)$ ou l'on va vers les ressources communautaires $(21,4 \%)$. Lorsqu'on a besoin de conseils sur l'impôt et la succession, on a d'abord recours aux proches (25\%), puis aux ressources communautaires $(16,2 \%)$, à un employé (15,6\%), aux professionnels du CLSC (12,3\%), ou l'on se débrouille seul (14,3\%). Quand on a des problèmes de relations avec le conjoint, on tente de les résoudre soi-même $(33,7 \%)$ ou l'on va vers les professionnels du CLSC $(20,8 \%)$ ou vers les proches $(10 \%)$ ou les ressources communautaires $(8,2 \%)$. Lorsqu'on éprouve des problèmes de relations avec les enfants, on fait appel aux mêmes ressources, sauf que dans cette situation on se fie davantage à soi-même $(38,2 \%)$ ou aux ressources communautaires $(11,1 \%)$. Quant aux problèmes de relations avec le propriétaire ou les locataires, on les règle soimême (38 \%) ou on va chercher l'aide des ressources communautaires $(15,4 \%)$ et parfois de professionnels du CLSC $(8,6 \%)$. Enfin, si l'on vit une situation d'abus ou d'exploitation de la part de l'entourage, on tente de s'en sortir soi-même $(27,6 \%)$ ou l'on a recours aux professionnels du CLSC (15,9\%), aux ressources communautaires $(15,5 \%)$ ou aux proches $(10,6 \%)$.

De plus, les ressources médicales sont mentionnées pour presque tous les problèmes. Ces ressources sont sans doute adéquates dans le cas de certaines difficultés, mais pas pour tous les problèmes. Ainsi, le médecin est identifié comme ressource potentielle par $8,7 \%$ des aînés inquiets pour leurs enfants et leur conjoint, par $8,1 \%$ de ceux qui ont besoin de conseils sur l'impôt et la succession, par $7,9 \%$ de ceux qui ont des relations problématiques avec leur conjoint, par $5,9 \%$ de ceux qui ont des difficultés avec leurs enfants et par $4,2 \%$ de ceux qui vivent des situations d'abus ou d'exploitation de la part de l'entourage. De toute évidence, les médecins sont à la fois des conseillers et des confidents pour nombre d'aînés, phénomène constaté antérieurement (Barker, Pistrang, Shapiro et Shaw, 1990). Mais cela ne veut pas dire que les sujets de l'étude sont indûment orientés vers les ressources médicales. Au contraire, même pour les inquiétudes face à la santé, plus du tiers des répondants $(34,9 \%)$ sont orientés vers eux-mêmes et leurs proches, ressources qui ne sont pas toujours appropriées en de telles circonstances. 


\section{Corrélations simples}

Les variables corrélées significativement aux orientations vers Soi-même, les Proches et les Services sont présentées au tableau 4. L'examen de ce tableau révèle plusieurs phénomènes intéressants. Premièrement, peu de variables sont significativement corrélées aux orientations de recherche d'aide et la plupart des corrélations significatives sont faibles. Ainsi, il n'existe pas de corrélation entre le nombre de Problèmes rencontrés et les orientations de recherche d'aide. Toutefois, pour six problèmes sur quinze (entretien de l'extérieur et de l'intérieur du domicile, transport, inquiétude par rapport à la santé, difficultés financières, besoin de conseils sur l'impôt et sur la succession), les aînés qui ont éprouvé la difficulté ont des orientations de recherche d'aide significativement différentes de ceux qui n'y ont pas été confrontés ${ }^{9}$. L'influence des problèmes sur les orientations de recherche d'aide serait donc limitée et contextuelle. Auquel cas, les orientations de recherche d'aide seraient peu sensibles aux aléas de la vie courante, parce que ce sont des prédispositions stables chez les individus.

Deuxièmement, il existe peu de liens entre les orientations de recherche d'aide et l'utilisation des services. Les corrélations entre les orientations de recherche d'aide et les Consultations médicales préventives et curatives ne sont pas significatives. Seule subsiste une corrélation faible entre l'orientation vers les Proches et le nombre d'Organismes de services contactés au cours des six mois ayant précédé l'étude. Cela laisse croire que les relations entre les orientations de recherche d'aide et l'utilisation des services sont médiatisées par les besoins de la personne.

En troisième lieu, le fait que le nombre de Services connus soit corrélé positivement à l'orientation vers les Services et négativement à l'orientation vers Soi-même corrobore la proposition voulant que les orientations de recherche d'aide aient une influence sur l'assimilation des informations relatives aux services. Tout se passe comme si l'orientation vers les Services amenait les

$9 \quad$ Les analyses statistiques (chi carré) qui fondent cette assertion ne sont pas présentées ici, mais sont disponibles auprès des auteurs. 
personnes à s'intéresser davantage aux ressources disponibles dans leur milieu. À l'inverse, les gens orientés vers eux-mêmes dans la solution de leurs problèmes accordent peu d'attention aux services.

Au reste, les corrélations simples vont dans le sens des hypothèses de l'étude et de la documentation consultée. Ainsi, la majorité des gens orientés vers eux-mêmes sont des hommes vivant en couple qui ne parlent guère de leurs problèmes et qui sont membres de peu de groupes d'aînés. Les gens orientés vers leurs proches ont davantage d'enfants, d'aidants en cas d'urgence et de contacts sociaux. Enfin, les personnes orientées vers les services sont moins âgées, plus souvent mariées, disposent de meilleurs revenus et ont une scolarité plus élevée. Ces personnes font probablement partie d'un univers culturellement proche de celui des dispensateurs de services. Cependant, la majorité des aînés orientés vers les services sont membres de groupes à faible cohésion. La corrélation considérée ici est faible mais significative. Dans la mesure où elle n'est pas le fruit du hasard, elle laisse croire que les membres des groupes d'aînés à forte cohésion sont moins orientés vers les services. Entretient-on des attitudes défavorables aux services dans ces groupes? II faudrait effectuer des recherches plus poussées pour le savoir. 


\section{Tableau 4}

Corrélations simples ( $r$ de Pearson) significatives entre certaines variables mesurées dans le questionnaire et les orientations de recherche d'aide

\begin{tabular}{|c|c|c|c|}
\hline & $\begin{array}{l}\text { Orientation } \\
\text { vers soi }\end{array}$ & $\begin{array}{c}\text { Orientation } \\
\text { vers les proches }\end{array}$ & $\begin{array}{c}\text { Orientation } \\
\text { vers les services }\end{array}$ \\
\hline Groupes de cohésion & 0,04 & $-0,02$ & $-0,144^{*}$ \\
\hline $\begin{array}{l}\text { Discussion de } \\
\text { problèmes }\end{array}$ & $-0,146^{\star *}$ & 0,06 & 0,10 \\
\hline $\begin{array}{l}\text { Nbre de services } \\
\text { connus }\end{array}$ & $-0,139 * *$ & 0,07 & $0,334^{* * *}$ \\
\hline Âge & $-0,01$ & $-0,06$ & $-0,262^{*}$ \\
\hline Nbre d'enfants & 0,001 & $0,136^{* *}$ & $-0,04$ \\
\hline $\begin{array}{l}\text { Sexe } \\
(0=F ; 1=H)\end{array}$ & $0,204^{* * *}$ & $-0,10$ & $-0,05$ \\
\hline $\begin{array}{l}\text { État civil } \\
(0=\text { non marié; } 1=\text { marié })\end{array}$ & $0,13^{*}$ & $-0,04$ & $0,145^{\star *}$ \\
\hline Revenu & 0,02 & $-0,10$ & $0,172^{* \star}$ \\
\hline Scolarité & $-0,06$ & $-0,03$ & $0,18^{* \star *}$ \\
\hline Nbre d'aidants & 0,03 & $0,218^{* * *}$ & 0,03 \\
\hline Contacts sociaux & $-0,07$ & $0,133^{*}$ & $-0,07$ \\
\hline $\begin{array}{l}\text { Nombre de groupes } \\
\text { fréquentés }\end{array}$ & $-0,147^{* *}$ & 0,04 & 0,05 \\
\hline $\begin{array}{l}\text { Nbre d'organismes } \\
\text { contactés }\end{array}$ & $-0,10$ & $0,106^{*}$ & 0,07 \\
\hline
\end{tabular}

${ }^{*} p<0,05 \quad{ }^{* *} p<0,01 \quad{ }^{* * *} p<0,001$

Sans astérisque : pas de corrélation significative.

\section{Vérification systématique des hypothèses}

Le tableau 5 présente les résultats des analyses discriminantes effectuées sur chacune des orientations de recherche d'aide. Tout d'abord, quand on compare les fonctions discriminantes, on constate qu'elles sont toutes statistiquement significatives. Toutefois, la proportion de variance expliquée $\left(R^{2}\right)$ est plus petite pour l'orientation vers Soi-même $(9,95 \%)$ que pour l'orientation vers les Proches (10,5\%) et pour l'orientation vers les Services $(32,4 \%)$. Cela signifie que les attributs considérés dans cette étude sont plus pertinents pour cette troisième orientation que pour les deux autres. Aussi, plus les fonctions sont discriminantes, plus grande 
est la proportion de sujets correctement classés, et c'est par rapport à l'orientation vers les Services que les sujets sont le mieux classés.

Le tableau 5 montre ensuite que les différentes orientations de recherche d'aide ont peu d'attributs communs. En fait, aucune variable ne fait partie des trois fonctions discriminantes, et une seule variable - le nombre de Services connus - est associée à deux orientations de recherche d'aide (vers Soi-même et vers les Services). Cela évoque le caractère singulier des orientations de recherche d'aide. Chacune d'elles ressortit à sa logique propre, comme le suggérait le cadre théorique.

Au regard des hypothèses, le tableau 5 montre que l'orientation vers Soi-même renvoie principalement à la méconnaissance des services et au fait d'être un homme. Les autres attributs sont de moindre importance. II semble que les gens fortement orientés vers eux-mêmes soient en rupture de ban avec l'univers des services, comme le veut probablement l'ethos de leur groupe de référence, en l'occurrence celui des hommes âgés (Dulac, 1997). Toutefois, ces gens les utilisent autant que les individus qui ont d'autres orientations. On peut donc recourir aux services sociaux et de santé par nécessité, sans s'y intéresser outre mesure.

Le profil des gens fortement orientés vers leurs Proches est différent. La fonction discriminante qui les distingue de leurs homologues faiblement orientés vers ce type de ressources est dominée par le nombre d'Aidants en cas d'urgence, suivi du nombre d'Enfants, de la fréquence des Contacts sociaux et du nombre d'Organismes contactés. Les gens fortement orientés vers leurs Proches sont insérés dans un milieu solidaire qui les dirige vers les services quand il ne peut leur fournir l'aide dont ils ont besoin. Rien ne permet de croire qu'une partie de ces gens soient issus d'un univers social réfractaire aux services, comme le suggérait la seconde hypothèse. Cependant, nous avons exclu de ces analyses les gens modérément orientés vers leurs proches qui appartiennent peut-être à un tel univers, assertion qui reste à vérifier.

Enfin, les aînés fortement orientés vers les Services se caractérisent par leur meilleure connaissance de ce type de ressources, 
par le fait qu'ils sont moins âgés et, de façon secondaire, par leur appartenance à un groupe à faible cohésion ainsi que par des revenus plus élevés. On peut croire qu'il s'agit de jeunes retraités de la classe moyenne qui sont des familiers de la sphère des services et qui s'y intéressent parce qu'ils prévoient en avoir besoin. Mais ces gens n'utilisent pas plus les services que leurs homologues peu orientés vers ces ressources ou axés sur d'autres formes d'aide. Dès lors, le fait d'être orienté vers les Services renvoie à au moins une réalité: l'ethos des classes favorisées et scolarisées. Utiliser les services est une réalité différente - qui est tributaire des besoins - car les orientations de recherche d'aide ne sont pas des comportements. 


\section{Tableau 5}

Tableau synthèse des analyses discriminantes effectuées sur les variables significativement corrélées aux orientations de recherche d'aide (coefficients standardisés)

\begin{tabular}{|c|c|c|c|}
\hline & $\begin{array}{l}\text { Orientation } \\
\text { vers soi }\end{array}$ & $\begin{array}{c}\text { Orientation } \\
\text { vers les } \\
\text { proches }\end{array}$ & $\begin{array}{c}\text { Orientation } \\
\text { vers les services }\end{array}$ \\
\hline Groupes de cohésion & $\mathrm{nc}$ & $\mathrm{nc}$ & $-0,40$ \\
\hline $\begin{array}{l}\text { Discussion de } \\
\text { problèmes }\end{array}$ & 0,275 & $\mathrm{nc}$ & $\mathrm{nc}$ \\
\hline $\begin{array}{l}\text { Nbre de services } \\
\text { connus }\end{array}$ & 0,515 & nc & 0,789 \\
\hline Âge & $\mathrm{nc}$ & $\mathrm{nc}$ & $-0,455$ \\
\hline Nbre d'enfants & $\mathrm{nc}$ & 0,489 & $\mathrm{nc}$ \\
\hline $\begin{array}{l}\text { Sexe } \\
(0=F ; 1=H)\end{array}$ & $-0,486$ & $\mathrm{nc}$ & $\mathrm{nc}$ \\
\hline $\begin{array}{l}\text { État civil } \\
(0=\text { non marié; } 1=\text { marié) }\end{array}$ & $-0,176$ & nc & $-0,097$ \\
\hline Revenu & $\mathrm{nc}$ & $\mathrm{nc}$ & 0,281 \\
\hline Scolarité & $\mathrm{nc}$ & $\mathrm{nc}$ & 0,062 \\
\hline Nbre d'aidants & $\mathrm{nc}$ & 0,717 & $\mathrm{nc}$ \\
\hline Contacts sociaux & $\mathrm{nc}$ & 0,207 & $\mathrm{nc}$ \\
\hline $\begin{array}{l}\text { Nombre de groupes } \\
\text { fréquentés }\end{array}$ & 0,264 & $\mathrm{nc}$ & $\mathrm{nc}$ \\
\hline $\begin{array}{l}\text { Nbre d'organismes } \\
\text { contactés }\end{array}$ & $\mathrm{nc}$ & 0,097 & $\mathrm{nc}$ \\
\hline $\begin{array}{l}\text { Centroïde du gr. } \\
\text { faiblement orienté }\end{array}$ & 0,329 & $-0,344$ & $-0,894$ \\
\hline $\begin{array}{l}\text { Centroïde du gr. } \\
\text { fortement orienté }\end{array}$ & $-0,332$ & 0,337 & 0,529 \\
\hline Corr. canonique & 0,3155 & 0,3238 & 0,5694 \\
\hline $\mathbf{R}^{2}$ & $9,95 \%$ & $10,5 \%$ & $32,4 \%$ \\
\hline Lambda de Wilks & $0,90^{* *}$ & $0,895^{* * *}$ & $0,676^{\star \star *}$ \\
\hline $\begin{array}{l}\text { \% d'individus classés } \\
\text { correctement }\end{array}$ & 62,3 & 64,7 & 75,7 \\
\hline n faible/fort & $100 / 99$ & $101 / 103$ & $55 / 93$ \\
\hline
\end{tabular}

** $p<0,01 \quad{ }^{* * *} p<0,001$

nc : Variable non considérée dans l'analyse.

\section{CONCLUSION ET DISCUSSION}

Dans cet article, nous avons étudié les orientations de recherche d'aide chez les aînés. Les résultats de la recherche présentée ont 
révélé le caractère distinct mais non exclusif des orientations de recherche d'aide ainsi que leur singularité. Leur caractère distinct tient au fait que les gens axés sur un type de ressources ne sont pas orientés sur d'autres formes d'aide. Par contre, les orientations vers les différents services sociaux et de santé sont corrélées entre elles, laissant croire que certains services peuvent se substituer les uns aux autres.

Le caractère non exclusif des orientations de recherche d'aide est mis en évidence par la pluralité des ressources mentionnées. Les gens semblent adopter une orientation principale qui renvoie à leur manière usuelle de réagir face aux difficultés de la vie, mais ils peuvent se tourner vers d'autres ressources quand les circonstances l'exigent. Les orientations de recherche d'aide subsidiaires sont des attitudes apprises et des habitus intériorisés qui permettent l'adaptation aux situations problématiques. Reste à savoir si les orientations subsidiaires sont aussi stables que l'orientation dominante ou principale. Le cas échéant, les orientations dominantes et subsidiaires seraient structurées de manière analogue aux activités pivots et contingentes (Delisle, 1982), et seraient des marqueurs de l'identité sociale des gens (Kelly, 1995).

Quant au caractère singulier des orientations de recherche d'aide, il est ressorti de la comparaison systématique des gens fortement et faiblement orientés vers chaque type de ressources. Les individus fortement axés sur les différentes ressources appartiennent à des univers socioculturels particuliers. Cet univers génère des attitudes spécifiques quant à la façon de résoudre les problèmes et inculque aux gens des habitus qui modulent leur comportement face aux difficultés. Ce qui surprend ici et qui nécessiterait un approfondissement, c'est que chaque orientation renvoie à un type de groupe différent: le groupe de sexe pour l'orientation vers Soi-même; les groupes primaires pour l'orientation vers les Proches et la classe socioéconomique et culturelle pour ce qui est de l'orientation vers les Services. Tout se passe comme si les gens étaient soumis à des influences sociales hétérogènes qui s'actualisent de façon hiérarchique dans des contextes donnés. En ce qui a trait à la recherche d'aide, il semble y avoir des circonstances où l'influence de l'ethos du principal groupe de référence cède le pas à un ethos subsidiaire associé à 
un groupe de référence de seconde importance (en temps normal) pour l'individu.

De plus, les résultats de notre étude indiquent qu'il y a peu de liens entre les orientations de recherche d'aide et l'utilisation des services. Cependant, l'orientation vers les services est le premier déterminant de leur connaissance, alors que les individus orientés vers eux-mêmes connaissent peu de ressources. Par conséquent, ceux qui sont orientés vers les services savent à qui s'adresser et le font probablement avec aisance. Inversement, on peut croire que les personnes non orientées vers les services les utilisent par nécessité ou sur recommandation de leurs proches, mais avec une certaine réticence. Ces individus profitent-ils autant des services reçus que les gens orientés vers les services? Notre recherche ne permet pas répondre de façon catégorique à cette question, mais ses résultats donnent à penser que les orientations de recherche d'aide ne sont pas tant déterminantes de la fréquence de l'utilisation des services que de la manière dont les usagers les abordent et, par extension, de la qualité des rapports entre les dispensateurs et les utilisateurs de services.

Cette conclusion interpelle les intervenants. Ceux-ci ont intérêt à déterminer l'orientation de recherche d'aide dominante de leurs clients pour mieux les servir. Car offrir des services à des gens qui désirent en recevoir suppose des stratégies différentes de celles qu'on doit élaborer pour rejoindre les personnes qui connaissent mal les services ou qui les perçoivent négativement. Les intervenants qui ont une idée juste des prédispositions de leurs clients face aux ressources mises à leur disposition peuvent donc plus facilement adapter leur offre de services et établir des relations de qualité avec leurs clients, à l'avantage de tous et chacun.

Marc-André DeLISLE

Professeur

Département de sociologie Université Laval

Hector OUELLET

Professeur

École de service social

Université Laval 


\section{Références bibliographique}

AuslandeR, G.K. et H. LITWIN (1990). "Social Support Networks and Formal Help Seeking: Differences Between Applicants to Social Services and a Nonapplicant Sample », Journal of Gerontoloy: Social Sciences, vol. 45, n 3, p. S112-119.

Barker, C., N. Pistrang, D.A. ShapiRo et I. Shaw (1990). « Coping and help-seeking in the UK adult population », British Journal of Clinical Psychology, vol. 29, n 3, p. 271-285.

BEAUd, J.-P. (1998). "Les techniques d'échantillonnage », dans B. Gauthier (dir.), Recherche sociale. De la problématique à la collecte des données. Québec, Presses de l'Université du Québec, p. 175200.

BÉLAND, F. (1982). Les principaux résultats de l'analyse des désirs d'hébergement de trois échantillons de personnes âgées du Québec, Québec, ministère des Affaires sociales du Québec.

BLAU, P.M. (1960a). "Structural effects », The American Sociological Review, vol. XXV, p. 178-193.

BLAU, P.M. (1960b). "A Theory of Social Integration », The American Journal of Sociology, vol. LXV, n 6, p. 545-556.

BoldUC, P. (1986). « Rapport de la recherche-action sur les services aux aînés ", dans R. Bernier (dir.), Le Troisième Âge: accès à l'information et utilisation des services, Sherbrooke, Éditions de l'Université de Sherbrooke, p. 27-114.

BourdieU, P. (1984). Questions de sociologie, Paris, Éd. de Minuit.

CALSYN, R.J. et L.A. RoAdes (1993). "Predicting Perceived Service Need, Service Awareness, and Service Utilization », Journal of Gerontological Social Work, vol. 21, nos 1-2, p. 59-76.

CANTOR, M.H. (1979). "Neighbors and Friends. An Overlooked Resource in the Informal Support System », Research on Aging, vol. 1, n 4, p. 434-463.

CANTOR, M.H. (1991). «Family and Community: Changing Roles in an Aging Society ", The Gerontologist, vol. 31, n 3, p. 337-346.

CANTOR, M.H. et V. LiTTLE (1985). " Aging and Social Care », dans R.H. Binstock et E. Shanas (dir.), Handbook of Aging and the Social Sciences, $2^{\mathrm{e}}$ éd., New York, Van Nostrand Reinhold, p. 745-781. 
ChAPpelL, N.L. (1985). "Social Support and the Receipt of Home Care Services », The Gerontologist, vol. 25, no 1, p. 47-54.

CHO, P.J. (1998). "Awareness and Utilization: A Comment», The Gerontologist, vol. 38, n 3, p. 317-319.

CoRIN, E.E. (1984). «Manière de vivre, manière de dire. Réseau social et sociabilité quotidienne des personnes âgées au Québec », dans Questions de culture, $n^{\circ} 6$, Québec, Institut québécois de recherche sur la culture, p. 157-186.

Corin, E.E., T. SHERIF et L. BERgeron (1983). Le fonctionnement des systèmes de support naturel, vol. 1, Québec, Laboratoire de gérontologie sociale de l'Université Laval.

CoWARD, R.T. (1988). "Aging in the Rural United States », dans E. Rathbone-McCuan et B. Havens, North American Elders. United States and Canadian Perspectives, New York, Grennwood Press, p. 161-178.

DelisLe, M.-A. (1982). "Loisir et structuration du temps chez les personnes âgées », Loisir et société, vol. 5, nº 2, p. 387-413.

DelisLe, M.-A. (1989), "Logiques sociales et soutien des personnes âgées », Service social, vol. 38, n 1, p. 102-121.

DelisLe, M.-A., N. Boucher et J. Roy (1993). " ... Pour s'entraider ». Étude portant sur la solidarité horizontale chez les personnes âgées du comté de Portneuf, Québec, Université Laval, Centre de recherche sur les services communautaires.

Delisle, M.-A. et H. Ouellet (2001). «Fréquentation des groupes d'aînés et recours aux services sociaux et de santé ", Revue canadienne du vieillissement / Canadian Journal on Aging, vol. 20, no 3, p. 307-337.

DULAC, G. (1997). «Plaidoyer pour une minorité oubliée: les hommes âgés ", Le Gérontophile, vol. 19, n 4, p. 3-9.

DURAND, J.-P. et R. WEIL (dir.) (1997). Sociologie contemporaine, $2^{\mathrm{e}}$ éd., Paris, Vigot.

GAUTHIER, H. et L. DUCHESNE (1991). Le vieillissement démographique et les personnes âgées au Québec, Québec, Les Publications du Québec.

GUIMOND, S. (1994). "Les groupes sociaux », dans R.J. Vallerand (dir.), Les fondements de la psychologie sociale, Boucherville, G. Morin Éditeur, p. 655-705. 
Hamelin, P. (1995). Un pionnier de l'entraide: le Service d'entraide Regroupement et Solidarité (Lévis-Métro) inc., Québec, IQRC (Institut québécois de recherche sur la culture).

Keating, N.C. (1991). Aging in Rural Canada, Toronto, Butterworths.

KeLlY, J.R. (1995). "Activities" dans G.L. Maddox (dir.), The Encyclopedia of Aging. A Comprehensive Resource in Gerontology and Geriatrics, $2^{\mathrm{e}}$ éd., New York, Springer Publ. Co., p. 4-7.

LAFRENAYE, Y. (1994). "Les attitudes et le changement des attitudes ", dans R.J. Vallerand (dir.), Les fondements de la psychologie sociale, Boucherville, G. Morin Éd., p. 327-405.

LALIVE D'ÉPINAY, CH. (1989). "Temps et classes sociales » dans G. Pronovost, D. Mercure et al., Temps et société, Québec, Institut québécois de recherche sur la culture, p. 223-258 (Coll. « Questions de culture »).

LASNIER, F. (1989). La mesure de la cohésion dans les équipes sportives, Sainte-Foy, Cégep de Sainte-Foy.

Lawton, M.P., M. Moss, C. Hoffman, R. Grant, T.T. Have et M.H. KLEBAN (1999). " Health, Valuation of Life, and the Wish to Live ", The Gerontologist, vol. 39, no 4, p. 406-415.

LEBEL, B. (1986). "Les relations entre les membres des communautés culturelles et les services sociaux et de santé », Canadian Ethnic Studies, vol. XVIII, nº 2, p. 79-89.

LITWAK, E. (1989). " Forms of Friendships Among Older People in an Industrial Society », dans R.G. Adams et R. Blieszner (dir.), Older Adult Friendship. Structure and Process, Newbury Park, CA, Sage Publ., p. 45-88.

LogAN, J.R. et G. SPITZE (1994). "Informal Support and the Use of Formal Services by Older Americans », Journal of Gerontology: Social Sciences, vol. 49, no 1, p. S25-34.

LuX, A. (1995). "Vieillissement démographique et réorganisation sociale » dans H. Gérard et V. Piché (dir.), La sociologie des populations, Montréal, PUM/AUPELF/UREF, p. 463-480.

MCCASLIN, R. (1989). "Service Utilization by the Elderly: The Importance of Orientation to the Formal System », Journal of Gerontological Social Work, vol. 14, nos 1-2, p. 153-174. 
MCKINLAY, J.B. (1972). "Some Approaches and Problems in the Study of the Use of Services - An Overview », Journal of Health and Social Behavior, vol. 13, p. 115-152.

MCKINLAY, J.B. (1973). "Social Networks, Lay Consultation and HelpSeeking Behavior », Social Forces, vol. 51, p. 275-292.

MitCheLL, J. et J.A. KROUT (1998). « Discretion and Service Use Among Older Adults : The Behavioral Model Revisited », The Gerontologist, vol. 38, no 2, p. 159-168.

MoEn, E. (1978). "The Reluctance of the Elderly to Accept Help ", Social Problems, vol. 25, n 3, p. 293-303.

Moon, A., J.E. LUBben et V. VILLA (1998). « Awareness and Utilization of Community Long-Term Care Services by Elderly Korean and NonHispanic White Americans ", The Gerontologist, vol. 38, $n^{\circ} 3$, p. 309-316.

MUDRACK, P.E. (1989). «Defining Group Cohesiveness. A Legacy of Confusion? ", Small Group Behavior, vol. 20, n 1, p. 37-49.

Mutran, E. et K.F. Ferraro (1988). «Medical Need and Use of Services Among Older Men and Women », Journal of Gerontology : Social Sciences, vol. 43, n 5, p. S162-171.

OuelLet, H. (1993). « Les orientations de recherche d'aide et la langue parlée », Service, social, vol. 42, n 1, p. 121-143.

PAQUET, M. (1996). «La réticence des personnes soutien de personnes âgées dépendantes vis-à-vis l'utilisation des services de soutien formels : un défi pour le milieu de la recherche et de l'intervention », Revue canadienne du vieillissement / Canadian Journal on Aging, vol. $15, n^{\circ} 3$, p. 442-462.

PenNing, M.J. (1990). «Receipt of Assistance by Elderly People: Hierarchical Selection and Task Specificity », The Gerontologist, vol. $30, n^{\circ} 2$, p. $220-227$.

PenNing, M.J. et L.A. Strain (1990). «Gender Differences in Disability, Assistance, and Subjective Well-Being in Later-Life », Journal of Gerontoloy : Social Sciences, vol. 49, nº 4, p. S202-208.

QUÉBEC [Prov.] (1993). La situation démographique au Québec (Louis Duchesne), Québec, Les Publications du Québec.

SANTÉ QUÉBEC (1995). Et la santé, ça va en 1992-1993? Rapport de l'enquête sociale et de santé 1992-1993, vol. 1, Montréal, ministère de la Santé et des Services sociaux, Gouvernement du Québec. 
Simard, C., M.-A. Delisle, D. Drouin et R. LaRouche (1992). Rapport d'étape, Projet de recherche CREDO du CLSC Arthur-Caux, SainteFoy et Laurier Station, Département d'éducation physique de I'Université Laval et CLSC Arthur-Caux, tomes 1, 2 et 3.

StOLLER, E.P. (1989). "Formal Services and Informal Helping: The Myth Of Service Substitution », The Journal of Applied Gerontology, vol. 8, no 1, p. 37-52.

TABACHNICK, B.G. et B.G. FIDELL (1996). Using Multivariate Statistics, $3^{\mathrm{e}}$ éd., New York, HarperCollins College Publ.

TIJHUIS, M.A.R., L. Peters et M. Foets (1990). « An Orientation toward Help-seeking for Emotional Problems », Social Science and Medicine, vol. 31, no 9, p. 989-995.

TREMBLAY, S. (1999). La pauvreté silencieuse : les conditions de vie des femmes âgées seules et pauvres de la Chaudière-Appalaches, Sainte-Marie, Régie régionale de la santé et des services sociaux de Chaudière-Appalaches.

WAN, T.T.H. (1989). " The Behavioral Model of Health Care Utilization by Older People » dans M.G. Ory et K. Bond (dir.), Aging and Health Care. Social Science and Policy Perspectives, New York et Londres, Routledge, p. 52-77.

WEINERT, C. et K.A. LONG (1987). "Understanding the Health Care Needs of Rural Families », Family Relations, vol. 36, p. 450-455.

WolinSKY, F.D. et R.J. JOHNSON (1991). « The Use of Health Services by Older People », Journals of Gerontology: Social Sciences, vol. 46, $n^{\circ} 6$, p. S345-357.

ZAY, N. (1981). Dictionnaire-manuel de gérontologie sociale, Québec, Presses de l'Université Laval. 